\title{
ALZHEIMERDEMENZ
}

\section{Das Gehirn schrumpft schon früh}

Nahezu ein Jahrzehnt bevor sich die ersten Symptome der Demenz zeigen, beginnen bestimmte Gehirnregionen bei Morbus Alzheimer zu schrumpfen, berichten Forscher der Harvard Medical School in Boston. Mittels Magnetresonanztomografie hatten sie insgesamt 56 Personen im Alter über 70 Jahren untersucht und bis zu elf Jahre nachbeobachtet. Dabei konzentrierten sich die Wissenschaftler auf die Hirnregionen, die mit Alzheimer in Zusammenhang gebracht werden wie etwa den Hippocam- pus. Von den Probanden mit der kleinsten Hirnmasse in diesen Bereichen erkrankten im Verlauf 55\% an Alzheimer, von den Teilnehmern mit der größten Hirnmasse in den betreffenden Gebieten entwickelte keiner eine Demenz. Personen mit durchschnittlicher Hirnmasse entwickelten zu 20\% Alzheimersymptome. Möglicherweise können NMR-Untersuchungen künftig die Alzheimer-Frühdiagnostik erleichtern. Neurology vom 13.4.2011

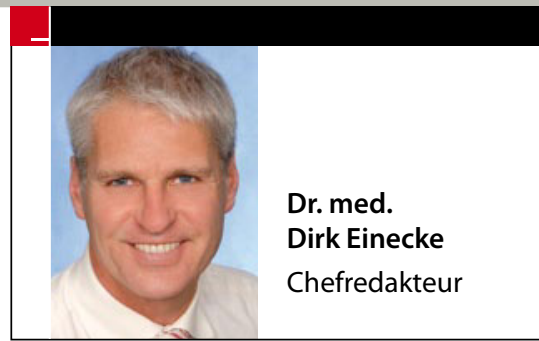

TYP-2-DIABETES BEI MÄNNERN

\section{Kann Testosteron das Leben verlängern?}

Typ-2-Diabetiker mit niedrigem Testosteronspiegel haben ein besonders hohes Sterberisiko. Dies ergab eine Studie der University Sheffield. Dort wurden 587 Männer mit Typ-2-Diabetes sechs Jahre lang beobachtet. 338 der Probanden hatten normale Testosteronspiegel, 182 erniedrigte Spiegel (unter $10,4 \mathrm{nmol} / \mathrm{l})$. Weitere 58 Probanden mit ebenfalls erniedrigtem Testosteronspiegel wurden mit dem Hormon substituiert. Im Studienverlauf verstarben 20\% der Probanden mit dem erniedrigten Testosteron, die nicht substituiert worden waren. In der Gruppe der Männer mit normalem Hormonspiegel verstarben $9 \%$, bei denen, die testosteronsubstituiert wurden, $8,6 \%$. Testosteronmangel ist bei Typ-2-Diabetikern häufig. Ob die Hormonsubstitution bei diesen Männern tatsächlich das Leben verlängert, muss in weiteren Studien geprüft werden.

Jahrestreffen der Society for Endocrinology in Birmingham, 13.4.2011

korrigieren lässt.

Jahresstatistik der American Society of Plastic Surgeons

\section{ANTICHOLINERGIKUM ODER BETAMIMETIKUM}

\section{Was schützt besser vor COPD-Exazerbationen?}

Als Medikamente der ersten Wahl werden für COPD-Patienten inhalative lang wirksame Bronchodilatatoren empfohlen. Ob dafür ein Anticholinergikum oder ein Betamimetikum besser geeignet ist, war bisher nicht bekannt. In der ersten Vergleichsstudie schnitt jetzt das Anticholinergikum bes- ser ab. Gegenüber Salmeterol verlängerte Tiotropium die Zeit bis zur ersten Exazerbation um 20\% (145 vs. 187 Tage). An der randomisierten Doppelblindstudie hatten 7376 COPD-Patienten teilgenommen.

N Engl J Med 2011;364:1093-1103; DGP-Jahrestagung, Dresden, 7.-10. 4.2011
www.SpringerMedizin.de

"Downloadcenter" unterstützt Ihre Arbeit

Im „Downloadcenter" finden Sie wichtige Helferlein für Ihre tägliche Praxis: z.B. ein Leichenschauprotokoll oder die MMW-Patienteninformationen zu häufigen Krankheitsbildern als PDF zum Mitgeben: www. springermedizin.de/downloadcenter. Also nichts wie rein ins Netz! 